\title{
New records of lichens from Manipur State, North-eastern India
}

\author{
Devi RKS ${ }^{1}$, Rout $\mathrm{J}^{1^{*}}$, Upreti DK ${ }^{2}$, Nayaka $\mathrm{S}^{2}$ and Pinokiyo $\mathrm{A}^{3}$ \\ ${ }^{I}$ Department of Ecology and Environmental Science, Assam University, Silchar-788011, Assam, India. \\ ${ }^{2}$ CSIR, National Botanical Research Institute, Rana Pratap Marg, Lucknow-226001,Uttar Pradesh, India \\ ${ }^{3}$ Department of Botany, D.M. College of Science, Imphal, Manipur-795001, India
}

Devi RKS, Rout J, Upreti DK, Nayaka S, Pinokiyo A 2015 - New records of lichens from Manipur State, North-eastern India. Mycosphere 6(6), 796-813, Doi 10.5943/mycosphere/6/6/13

\begin{abstract}
A total of 140 lichen species belonging to 50 genera and 23 families with 39 species recorded for the first time from the state of Manipur, Northeast India, being reported. Fourteen species are new additions to the lichen flora of Northeast India. The crustose morphotype (49\% of the species found) dominated the area, followed by foliose (43\%), dimorphic, fruticose, leprose and squamulose lichens, each contributing by $4 \%, 2 \%, 1 \%$ and $1 \%$ of the species found, respectively. Nine species of phorophytic foliicolous lichens that usually colonize live leaves were also encountered. The occurrence of foliicolous lichens is quite characteristic of species diversity and indicate rather high richness in this relatively small phytogeographical area. A brief note on the phytogeographic affinities and distribution of the newly recorded lichens in Manipur has been provided.
\end{abstract}

Key words - Crustose - Foliicolous - Parmeliacaea - Parmotrema

\section{Introduction}

India is considered the fifth country with the greatest biodiversity in the world, with about $10 \%$ of the 20,000 species of lichens recorded in the world (Groombridge 1992). About 2400 lichen species distributed on 305 genera and 74 families have been reported to occur on various substrata in tropical, subtropical, temperate and alpine regions of India (Singh \& Sinha 2010). Western Ghats is the richest State with ca. 800 species (39\% ot currently known species), followed by Eastern Himalayas with 759 species $(37 \%)$ and the Western Himalayas with 550 species (27 $\%)$. The dominant families are Parmeliaceae, Graphidaceae, Physciaceae, Usneaceae, Cladoniaceae, while the dominat genera are Parmelia, Graphina, Usnea, Graphis and Lecanora . About $23 \%$ of the known species, mainly belonging to Graphina, Trypethelium, Graphis and Porina, are supposed to be endemic to India. Apart from their use as bioindicators of air pollution, lichens in India are known for a variety of uses, such as spices, flavouring agents, medicines and edible. Over 30 species are used in perfumery industry owing to the presence of aromatic resinoids.

Despite such a high diversity of lichen flora (Awasthi 1960), those belonging to Northeast India, in particular, have not been adequately explored. The landscape of Manipur, one of the seven States of this region, consists of rugged hillocks, narrow valleys, and flat plains. Although lichens from Manipur were recorded as early as 1892 (Müll. Arg. 1892), more than a century has passed 


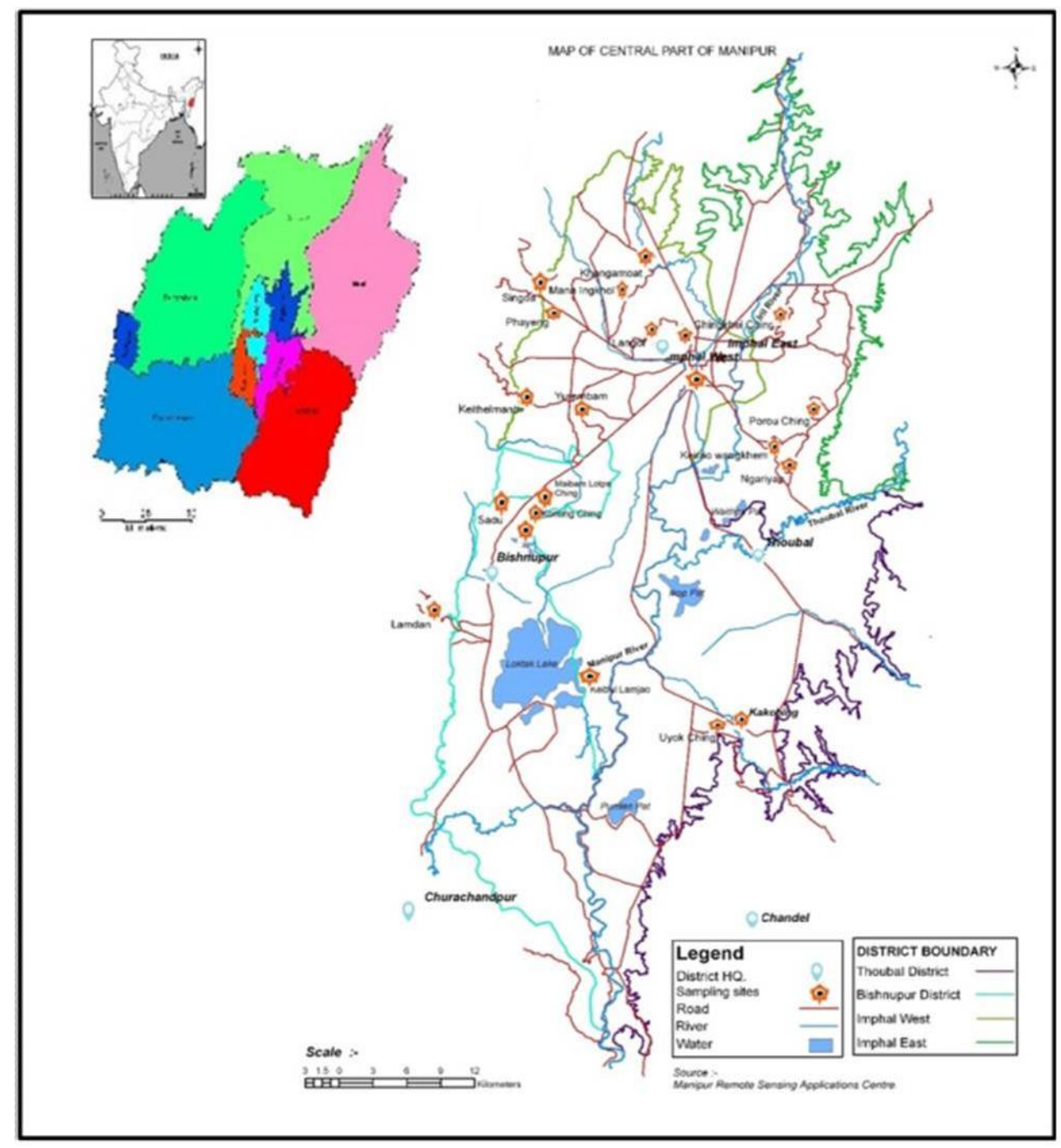

Fig.1 - Map of Manipur state showing the study sites

with only a few other sporadic reports (Awasthi 1960, 1987, Singh 1984, Singh 1980, 1981a, 1981b, 1983, 1984, Patwardhan \& Nagarkar 1982, Singh \& Upreti 1986, 1990, 1993) have appeared, the last one by Singh \& Pinokiyo in 2003. Following rapid urbanisation and anthropogenic activities in the recent past, and as a sequel to our continued effort to assess the lichen flora from Northeast India, 39 new species of lichens, are reported from the State of Manipur as first records. Also, fourteen of these species are completely new to the entire Northeast India region.

\section{Materials \& Methods}

This study is based on more than 1100 specimens of lichens collected from twenty localities of Manipur State $\left(23^{\circ} 83^{\prime}-25^{\circ} 68^{\prime} \mathrm{N}\right.$ and $\left.93^{\circ} 03^{\prime}-94^{\circ} 78^{\prime} \mathrm{E}\right)$, located in the Northeast part of India (Fig. 1). The specimens were collected from different substrata such as barks, twigs, rocks, soil and leaves. Trees (Bauhinia purpurea, Castanopsis hystrix, Ficus bengalensis, Mangifera indica, Magnolia pterocarpa, and Quercus lamellose) exhibit luxuriant growth of lichens. The identification of the specimens was done by studying their morphology, anatomy and chemistry. The morphology was studied using a LaboMed Digi Zoom dissecting microscope, and the anatomical details were studied using a Leica TM DM 500 optical microscope. Colour tests were performed using $\mathrm{KOH}(\mathrm{K})$, calcium hypochlorite $(\mathrm{C})$ and para-phenylenediamine $(\mathrm{P})$ solution. Secondary metabolites were identified by thin layer chromatography (TLC) as described by Walker \& James (1980). The chromatograms were done in solvent system A (toluene: dioxane: acetic acid: 
Table 1 Species composition of lichens in central part of Manipur

\begin{tabular}{|c|c|c|c|c|c|c|c|c|c|c|c|c|c|c|c|c|c|c|c|c|c|c|c|}
\hline & Species & Sub & GF & $\mathbf{C C}$ & $\mathbf{K N}$ & $\mathbf{K L}$ & KW & $\mathbf{K M}$ & KG & $\mathbf{K J}$ & $\mathbf{K C}$ & LD & LG & $\mathbf{L P}$ & ML & MI & NR & PY & PC & SC & SD & $\mathbf{U C}$ & $\overline{\mathbf{Y R}}$ \\
\hline \multicolumn{24}{|c|}{ Arthoniaceae } \\
\hline 1. & Arthonia inconspicua Stirt & B & $\mathrm{C}$ & & & & & & & & & & & & & & & & & & & & + \\
\hline 2. & $\begin{array}{l}\text { Arthonia tumidula (Ach.) } \\
\text { Ach. }\end{array}$ & $\mathrm{B}$ & $\mathrm{C}$ & & + & + & & & & & & & & + & & & & & + & + & & & \\
\hline 3. & $\begin{array}{l}\text { Arthothelium abnorme } \\
\text { (Ach.) Müll. Arg. }\end{array}$ & B & $\mathrm{C}$ & & & & + & & & & & & & & & & & & + & & & & \\
\hline 4. & $\begin{array}{l}\text { Arthothelium chiodectoides } \\
\text { (Nyl.) Zahlbr. }\end{array}$ & B & $\mathrm{C}$ & + & & & & & & + & & & & & & + & & & + & & & + & \\
\hline 5. & $\begin{array}{l}\text { Cryptothecia dissimilis } \\
\text { Makhija \& Patw. }\end{array}$ & $\mathrm{B}$ & $\mathrm{C}$ & & & & & & & & + & & & & & & + & & & + & & + & \\
\hline 5. & $\begin{array}{l}\text { Cryptothecia lunulata } \\
\text { (Zahlbr.) Makhija \& Patw. }\end{array}$ & B & $\mathrm{C}$ & + & & + & + & + & + & & + & + & & + & & & & & & + & & & \\
\hline 7. & $\begin{array}{l}\text { Cryptothecia multipunctata } \\
\text { Jagadeesh \& al. }\end{array}$ & B & $\mathrm{C}$ & & & & & & & & & & & & & & + & & & & & & \\
\hline 3. & $\begin{array}{l}\text { Herpothallon granulare } \\
\text { (Sipman) Aptroot \& } \\
\text { Lücking }\end{array}$ & $\mathrm{B}$ & $\mathrm{C}$ & & & & + & & + & + & + & & + & + & & & + & + & + & + & + & + & \\
\hline э. & $\begin{array}{l}\text { Herpothallon isidiatum } \\
\text { Jagadeesh \& G.P. Sinha }\end{array}$ & $\mathrm{B}$ & $\mathrm{C}$ & & & + & & + & + & & & + & & + & + & & + & + & & & & & + \\
\hline \multicolumn{24}{|c|}{ Caliciaceae } \\
\hline & $\begin{array}{l}\text { Baculifera curtisii (Tuck.) } \\
\text { Marbach }\end{array}$ & $\mathrm{B}$ & $\mathrm{C}$ & + & + & & + & & & & & + & + & & & & & & + & & + & + & \\
\hline \multicolumn{24}{|c|}{ Candelariaceae } \\
\hline & $\begin{array}{l}\text { Candelaria concolor } \\
\text { (Dicks.) Stein* }\end{array}$ & $\mathrm{B}$ & $\mathrm{F}$ & & & & & + & & & + & & + & + & + & & & & & + & & & + \\
\hline \multicolumn{24}{|c|}{ Chrysothricaceae } \\
\hline & $\begin{array}{l}\text { Chrysothrix candelaris (L.) } \\
\text { J.R. Laundon* }\end{array}$ & B & Le & & & + & & & & & & & & & & & & & & & & & \\
\hline \multicolumn{24}{|c|}{ Cladoniaceae } \\
\hline 13. & $\begin{array}{l}\text { Cladonia awasthiana Ahti \& } \\
\text { Upreti* }\end{array}$ & $S$ & $\mathrm{D}$ & & & & & & & & & & & & & & & & + & & & & \\
\hline 14. & $\begin{array}{l}\text { Cladonia macroceras } \\
\text { (Delise) Hav.** }\end{array}$ & $S$ & $\mathrm{D}$ & & & & & & + & & & & & & & & & & & & & & \\
\hline 15. & Cladonia rei Schaer* & $S$ & $\mathrm{D}$ & & & & & & & & & & & & & + & & & & & & & \\
\hline 16. & Cladonia sp. & $S$ & $\mathrm{D}$ & & & & & + & & & & & & & & & & & & & & & \\
\hline 17. & $\begin{array}{l}\text { Cladonia verticillata } \\
\text { (Hoffm) Shaer }\end{array}$ & $\mathrm{S}$ & $\mathrm{D}$ & & & & & & & & & & & & & + & & & & & & & \\
\hline
\end{tabular}


18. Coccocarpia palmicola

B F

(Spreng.) Arv. \& D.J.

Galloway

Collemataceae
19. Collema leptaleum var.
biliosum (Mont.) Degel.**
20. Collema pulcellum var.
subnigrescens (Müll.Arg.)
Degel.
21. Collema sp.
22. Leptogium
austroamericanum (Malme)
C.W. Dodge
23. eptogium cyanescens
(Rabenh.) Körb.
24. Leptogium denticulatum
Nyl.
25. Leptogium pichneum (Ach.)
Malme**
26. Leptogium
$\mathrm{B} / \mathrm{R} \quad \mathrm{F}$
B $\quad \mathrm{F}$
B F
B F

pseudopapillosum P.M.

Jørg.*

Ectolechiaceae

27. Lopadium leucoxanthum

(Spreng.) Zahlbr.

28. Sporopodium sp.

Graphidaceae

29. Diorygma junghuhnii (Mont.

\& Bosch) Kalb \& al

30. Diorygma megasporum Kalb \&

al.

31. Fissurina sp.

32. Graphis aurita Eschw.

33. Graphis caesiella Vain*

34. Graphis lineola Ach.

35. Graphis nigroglauca Leight.

36. Graphis pertricosa (Kremp.)

$+$

$+$

A.W. Archer

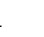

(K)

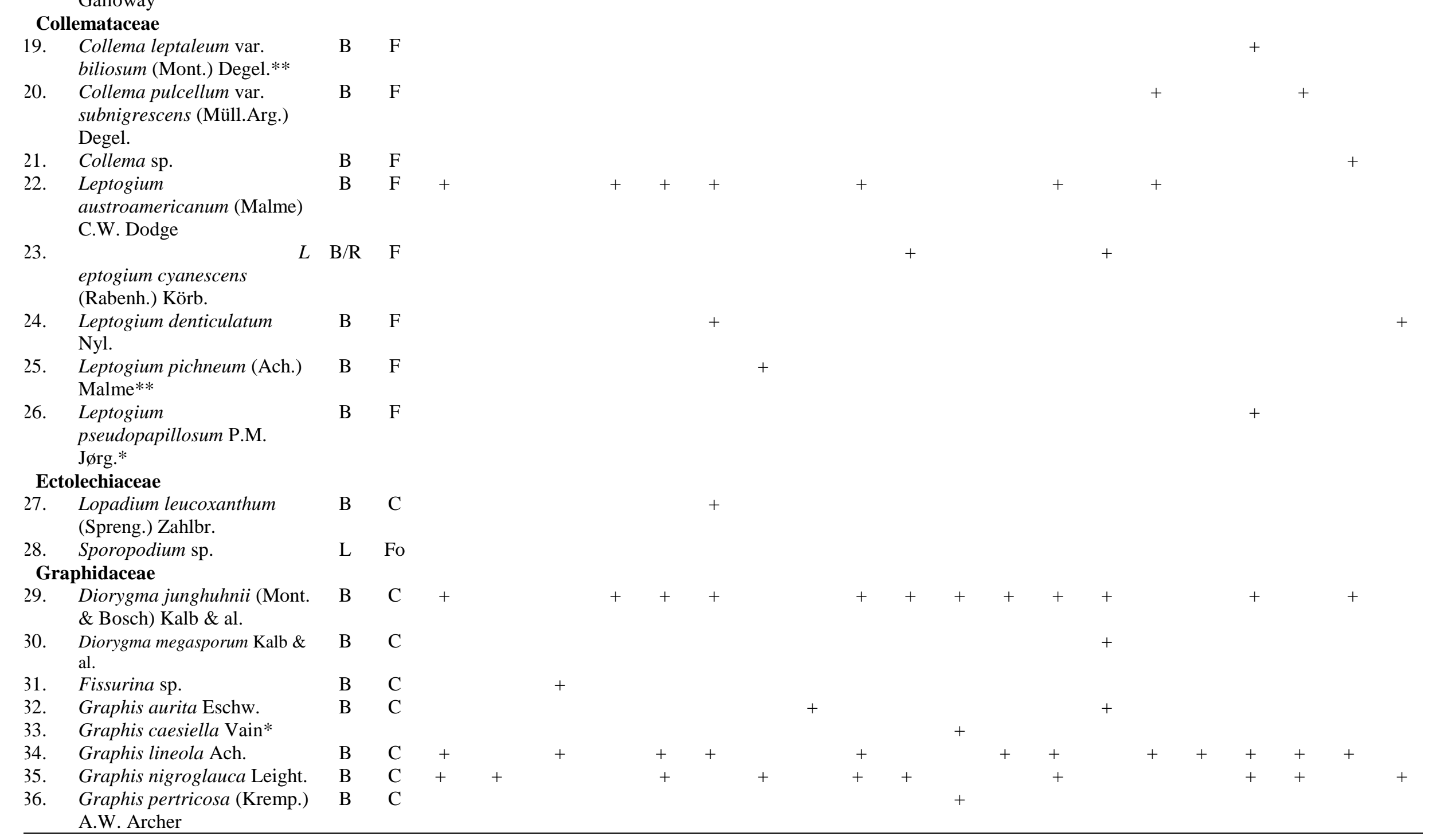




\begin{tabular}{|c|c|c|c|c|c|c|c|c|c|c|c|c|c|c|c|c|c|c|c|c|c|c|c|}
\hline & Species & Sub & GF & $\mathrm{CC}$ & $\mathbf{K N}$ & $\mathbf{K L}$ & KW & $\mathbf{K M}$ & KG & KJ & KC & LD & LG & $\mathbf{L P}$ & ML & MI & NR & PY & PC & SC & SD & $\mathbf{U C}$ & YR \\
\hline 37. & Graphis platycarpa Eschw. & $\mathrm{B}$ & $\mathrm{C}$ & + & & & + & & & & & & & & & & & & & & & & \\
\hline 38. & Graphis proserpens Vain. & B & $\mathrm{C}$ & & & & & & & & & & & & & & + & & & + & & & \\
\hline 39. & $\begin{array}{l}\text { Graphis pyrrhocheiloides } \\
\text { Zahlbr.*** }\end{array}$ & B & $\mathrm{C}$ & & & & & & & & & & & + & & & & & & & & & \\
\hline $\begin{array}{l}40 . \\
41 .\end{array}$ & $\begin{array}{l}\text { Graphis scripta (L.) Ach. } \\
\text { Graphis sp. }\end{array}$ & B & $\mathrm{C}$ & & & $\begin{array}{l}+ \\
+\end{array}$ & + & + & & & & & & + & + & & & & & & & & + \\
\hline 42. & $\begin{array}{l}\text { Graphis verminosa Müll. } \\
\text { Arg. }\end{array}$ & B & $\mathrm{C}$ & & & & & & & & & & & & & + & & & & & & & \\
\hline 43. & Phaeographis sp. & B & $\mathrm{C}$ & & & & & & & & & & & + & & & & & & & & & \\
\hline Ha & $\begin{array}{l}\text { Thecaria austroindica (D.D. } \\
\text { Awasthi \& Upreti) } \\
\text { Kr.P.Singh \& G.P. Sinha } \\
\text { matommataceae }\end{array}$ & B & $\mathrm{C}$ & & & + & & & & & & & & + & + & + & & & & + & & + & \\
\hline $\begin{array}{l}45 . \\
\text { Le }\end{array}$ & $\begin{array}{l}\text { Haematomma puniceum } \\
\text { (Sw.) A. Massal. } \\
\text { anoraceae }\end{array}$ & B & $\mathrm{C}$ & + & + & & + & & & & & + & & & + & + & + & & & + & & + & \\
\hline 46. & Lecanora achroa Nyl. & $\mathrm{B} / \mathrm{R}$ & $\mathrm{C}$ & + & + & + & & + & + & & & + & + & & + & + & + & + & & + & + & + & + \\
\hline 47. & $\begin{array}{l}\text { Lecanora japonica Müll. } \\
\text { Arg. }\end{array}$ & $\mathrm{B}$ & $\mathrm{C}$ & & & & & & & & & & & & & + & & & & & & + & \\
\hline $\begin{array}{ll}48 . \\
\text { Le }\end{array}$ & $\begin{array}{l}\text { Lecanora tropica Zahlbr. } \\
\text { ideaceae }\end{array}$ & B & $\mathrm{C}$ & + & & + & & & & & & + & & & & & + & & & + & & & \\
\hline 49. & $\begin{array}{l}\text { Lecidea granifera (Ach.) } \\
\text { Vain. }\end{array}$ & B & $\mathrm{C}$ & & & & + & & + & & & & & & + & & & & & & & + & \\
\hline & couitiaceae & & & & & & & & & & & & & & & & & & & & & & \\
\hline $\begin{array}{l}50 . \\
\mathrm{Ne}\end{array}$ & $\begin{array}{l}\text { Letrouitia transgressa } \\
\text { (Malme) Hafellner \& } \\
\text { Bellem. } \\
\text { hromataceae }\end{array}$ & $\mathrm{B}$ & $\mathrm{C}$ & & & & & & + & & & & & & & & & & & & & & \\
\hline & $\begin{array}{l}\text { Nephroma sikkimense } \\
\text { Asahina* }\end{array}$ & B & $\mathrm{F}$ & & & & & & & & & & & & & & & & & + & & & \\
\hline & meliacaea & & & & & & & & & & & & & & & & & & & & & & \\
\hline 52. & Bulbothrix isidiza (Nyl.) Hale & B & $\mathrm{F}$ & + & + & & + & + & & + & & + & + & & + & + & + & & + & + & + & & + \\
\hline 53. & $\begin{array}{l}\text { Bulbothrix setschwanensis } \\
\text { (Zahlbr.) Hale }\end{array}$ & B & $\mathrm{F}$ & & & & & + & & + & & & & & & & + & + & & & & & \\
\hline 54. & $\begin{array}{l}\text { Bulbothrix tabacina (Mont. } \\
\text { \& Bosch) Hale* }\end{array}$ & B & $\mathrm{F}$ & + & & & & & & & & & & & & + & + & + & & & & & \\
\hline 55. & $\begin{array}{l}\text { Canoparmelia texana } \\
\text { (Tuck.) Elix \& Hale }\end{array}$ & B & $\mathrm{F}$ & & & & + & & & & & & & & & & & & & & + & & \\
\hline 56. & $\begin{array}{l}\text { Hypotrachyna flexilis } \\
\text { (Kurok) Hale }\end{array}$ & B & $\mathrm{F}$ & & & & & & & & & & & & & & & & & + & & & \\
\hline
\end{tabular}




\begin{tabular}{|c|c|c|c|c|c|c|c|c|c|c|c|c|c|c|c|c|c|c|c|c|c|c|c|}
\hline & Species & Sub & GF & $\mathbf{C C}$ & $\mathbf{K N}$ & KL & KW & $\mathbf{K M}$ & KG & $\mathbf{K J}$ & KC & LD & LG & LP & ML & MI & NR & PY & PC & SC & SD & $\mathbf{U C}$ & YR \\
\hline$\overline{57 .}$ & $\begin{array}{l}\text { Hypotrachyna revoluta } \\
\text { (Flörke) Hale** }\end{array}$ & $\mathrm{B}$ & $\mathrm{F}$ & & & & + & & & & & & & & & & & & & & & & \\
\hline 58. & $\begin{array}{l}\text { Myelochroa aurulenta } \\
\text { (Tuck.) Hale }\end{array}$ & $\mathrm{B}$ & $\mathrm{F}$ & & & & & & & & & & & & & & & & & + & & & \\
\hline 59. & $\begin{array}{l}\text { Myelochroa xantholepis } \\
\text { (Mont. \& Bosch) Elix \& } \\
\text { Hale }\end{array}$ & B & $\mathrm{F}$ & & & & & & & & & & & & & & & & & & & + & \\
\hline 50. & $\begin{array}{l}\text { Parmelinella wallichiana } \\
\text { (Taylor) Elix \& Hale }\end{array}$ & B & $\mathrm{F}$ & & & & & & & + & & & & & & & & & & & & & \\
\hline 51. & $\begin{array}{l}\text { Parmotrema andinum (Müll. } \\
\text { Arg.) Hale** }\end{array}$ & B & $\mathrm{F}$ & & & & & & + & & & & + & & & & + & & & & & + & \\
\hline 52. & $\begin{array}{l}\text { Parmotrema austrosinense } \\
\text { (Zahlbr.) Hale }\end{array}$ & B & $\mathrm{F}$ & + & + & + & + & + & + & & + & + & + & + & + & + & + & + & & + & + & + & + \\
\hline 53. & $\begin{array}{l}\text { Parmotrema crinitum (Ach.) } \\
\text { M. Choisy** }\end{array}$ & B & $\mathrm{F}$ & & & & + & & + & & & & & & & & & & & + & & + & \\
\hline 54. & $\begin{array}{l}\text { Parmotrema cristiferum } \\
\text { (Taylor) Hale }\end{array}$ & B & $\mathrm{F}$ & & & & & + & & & & + & & & & & & & & & & + & \\
\hline 55. & $\begin{array}{l}\text { Parmotrema eunetum (Stirt.) } \\
\text { Hale** }\end{array}$ & B & $\mathrm{F}$ & & & & & & & + & + & & & & & & & & & & & & \\
\hline 56. & $\begin{array}{l}\text { Parmotrema hababianum } \\
\text { (Gyeln.) Hale }\end{array}$ & B & $\mathrm{F}$ & + & + & & & + & + & & & & & & & + & & & + & & & & + \\
\hline 57. & Parmotrema indicum Hale* & B & $\mathrm{F}$ & + & & & & & + & & & & + & & & + & + & & + & & & & \\
\hline 58. & $\begin{array}{l}\text { Parmotrema mellissii (C.W. } \\
\text { Dodge) Hale }\end{array}$ & B & $\mathrm{F}$ & & + & & & & & & & & & & & & & & & & & & \\
\hline 59. & $\begin{array}{l}\text { Parmotrema mesotropum } \\
\text { (Müll. Arg.) Hale* }\end{array}$ & $\mathrm{B} / \mathrm{R}$ & $\mathrm{F}$ & & & & & & & & & & & + & & & & & & & + & & \\
\hline 70. & $\begin{array}{l}\text { Parmotrema } \\
\text { praesorediosum (Nyl.) Hale }\end{array}$ & B & $\mathrm{F}$ & + & + & + & & & + & & & + & + & & & + & & + & & & & + & + \\
\hline 71. & $\begin{array}{l}\text { Parmotrema ravum (Krog \& } \\
\text { Swinscow) Sérus. }\end{array}$ & B & $\mathrm{F}$ & & & & & & & & & & & & & + & & & & & & & \\
\hline 72. & $\begin{array}{l}\text { Parmotrema reticulatum } \\
\text { (Taylor) M. Choisy }\end{array}$ & B & $\mathrm{F}$ & + & & & + & + & + & + & + & + & + & + & + & + & + & + & & + & & + & + \\
\hline 73. & $\begin{array}{l}\text { Parmotrema saccatilobum } \\
\text { (Taylor) Hale* }\end{array}$ & B & $\mathrm{F}$ & & & & & & & & & & & & & + & & & & & + & & + \\
\hline 74. & $\begin{array}{l}\text { Parmotrema subarnoldii } \\
\text { (Abbayes) Hale }\end{array}$ & B & $\mathrm{F}$ & + & & & & & & & & & & & & & & & & & & & \\
\hline 75. & $\begin{array}{l}\text { Parmotrema tinctorum } \\
\text { (Despr. ex Nyl.) Hale }\end{array}$ & $\mathrm{B}$ & $\mathrm{F}$ & + & + & & + & + & + & + & + & + & + & + & + & + & + & + & + & + & + & + & + \\
\hline 76. & Usnea pangiana Stirt.* & $\mathrm{B} / \mathrm{T}$ & FR & & & & & & & & & + & & & & & & & & & & & \\
\hline 77. & Usnea pectinata Taylor & $\mathrm{B}$ & FR & & & & & & & & & & & & & + & & & & & & & \\
\hline
\end{tabular}




\begin{tabular}{|c|c|c|c|c|c|c|c|c|c|c|c|c|c|c|c|c|c|c|c|c|c|c|c|}
\hline & Species & Sub & GF & $\mathbf{C C}$ & $\mathbf{K N}$ & $\mathbf{K L}$ & KW & $\mathbf{K M}$ & $\mathbf{K G}$ & $\mathbf{K J}$ & KC & LD & LG & $\mathbf{L P}$ & ML & MI & NR & PY & PC & SC & SD & $\mathbf{U C}$ & $\mathbf{Y R}$ \\
\hline \multicolumn{24}{|c|}{ Pertusariaceae } \\
\hline 78. & Pertusaria leioplacella Nyl. & B & C & & & & & & & & & & & & & & & & + & & & & \\
\hline 79. & $\begin{array}{l}\text { Pertusaria pseudococcodes } \\
\text { Müll. Arg. }\end{array}$ & $\mathrm{B}$ & $\mathrm{C}$ & & & & & & & & & + & & & + & & + & + & & & & & \\
\hline 30. & $\begin{array}{l}\text { Pertusaria tetrathalmia } \\
\text { (Fée) } \mathrm{Nyl}^{* *}\end{array}$ & B & $\mathrm{C}$ & & & & & & & & & & & & & & & & & & & & + \\
\hline \multicolumn{24}{|c|}{ Physciaceae } \\
\hline 31. & $\begin{array}{l}\text { Buellia morehensis Kr.P. } \\
\text { Singh \& S.R. Singh }\end{array}$ & B & $\mathrm{C}$ & & & & & & & & & & & + & & & & & & & & & \\
\hline 32. & $\begin{array}{l}\text { Dirinaria applanata (Fée) } \\
\text { D.D. Awasthi* }\end{array}$ & B & $\mathrm{F}$ & + & + & & & & + & & + & + & & & & & + & & + & + & & + & + \\
\hline 33. & $\begin{array}{l}\text { Dirinaria confluens (Fr.) } \\
\text { D.D. Awasthi* }\end{array}$ & B & $\mathrm{F}$ & & & + & & & & & & & & & & + & & & & & + & & \\
\hline 34. & $\begin{array}{l}\text { Dirinaria consimilis (Stirt.) } \\
\text { D.D. Awasthi }\end{array}$ & B & $\mathrm{F}$ & + & + & & & + & + & + & + & + & + & + & + & + & + & + & + & + & & + & + \\
\hline 35. & $\begin{array}{l}\text { Dirinaria picta }(\mathrm{Sw} .) \text { Clem. } \\
\& \text { Shaer* }\end{array}$ & B & $\mathrm{F}$ & & & & & & & & + & & & & + & + & + & & + & & & & \\
\hline 36. & $\begin{array}{l}\text { Heterodermia albidiflava } \\
\text { (Kurok.) D.D. Awasthi* }\end{array}$ & B & $\mathrm{F}$ & & & & + & & & & & & & & & + & + & & & & & & \\
\hline 37. & $\begin{array}{l}\text { Heterodermia diademata } \\
\text { (Taylor) D.D. Awasthi }\end{array}$ & B & $\mathrm{F}$ & + & + & & + & + & + & + & + & + & + & + & + & + & + & + & & & + & + & + \\
\hline 38. & $\begin{array}{l}\text { Heterodermia hypocaesia } \\
\text { (Yasuda) D.D. Awasthi }\end{array}$ & B & $\mathrm{F}$ & & & & + & & & & + & & & & & & + & + & & & & & \\
\hline 39. & $\begin{array}{l}\text { Heterodermia hypochraea } \\
\text { (Vain) Swinscow \& Krog* }\end{array}$ & B & $\mathrm{F}$ & + & & & + & & & & & & & & & & + & & & & & & \\
\hline 90. & $\begin{array}{l}\text { Heterodermia incana (Stirt.) } \\
\text { D.D. Awasthi }\end{array}$ & B & $\mathrm{F}$ & & & & & & & & & & & & & & + & & & & & & \\
\hline$\exists 1$. & $\begin{array}{l}\text { Heterodermia japonica (M. } \\
\text { Satô) Swinscow \& Krog }\end{array}$ & B & $\mathrm{F}$ & & & & + & & & & & & & & & & & & & & & & \\
\hline 92. & $\begin{array}{l}\text { Heterodermia obscurata } \\
\text { (Nyl.) Trevis. }\end{array}$ & B & $\mathrm{F}$ & & & & + & & & & & + & & & & + & + & & & & & & \\
\hline 93. & $\begin{array}{l}\text { Heterodermia pseudospeciosa } \\
\text { (Kurok.)W.L. Culb. }\end{array}$ & B & $\mathrm{F}$ & & & + & & & & & & + & & & & + & + & & & + & & + & \\
\hline 94. & Hyperphyscia sp. & B & $\mathrm{F}$ & + & & & & & & & & & & & & & & & & & & & \\
\hline 95. & $\begin{array}{l}\text { Phaeophyscia endococcina } \\
\text { (Körb.) Moberg* }\end{array}$ & $\mathrm{B}$ & $\mathrm{F}$ & & & & & & & & & & & & & & & & & + & & & \\
\hline 96. & $\begin{array}{l}\text { Phaeophyscia hispidula } \\
\text { (Ach.) Moberg }\end{array}$ & $\begin{array}{c}\mathrm{CW} / \\
\mathrm{M}\end{array}$ & $\mathrm{F}$ & & & & & & & & & & & & + & & & & & & & & \\
\hline 97. & $\begin{array}{l}\text { Pyxine berteriana (Fée) } \\
\text { Imshaug }\end{array}$ & B & $\mathrm{F}$ & & & & & & & & & & + & & & + & + & + & & & + & & \\
\hline
\end{tabular}




\begin{tabular}{|c|c|c|c|c|c|c|c|c|c|c|c|c|c|c|c|c|c|c|c|c|c|c|}
\hline Species & Sub & GF & $\mathrm{CC}$ & KN & KL & KW & $\mathbf{K M}$ & KG & KJ & KC & LD & LG & LP & ML & MI & NR & PY & $\mathbf{P C}$ & SC & SD & $\mathbf{U C}$ & YR \\
\hline Pyxine cocoes (Sw.) Nyl. & $\mathrm{B}$ & $\mathrm{F}$ & & + & + & & & + & & & & + & + & & & & & & & & + & \\
\hline $\begin{array}{l}\text { 99. Pyxine himalayensis D.D. } \\
\text { Awasthi** }\end{array}$ & B & $\mathrm{F}$ & & & & + & & & & & & & & & & & & & & & & \\
\hline 100. Pyxine petricola $\mathrm{Nyl} . *$ & $\mathrm{~B} / \mathrm{R}$ & $\mathrm{F}$ & & & & & & & & & & & & & & & + & & & & & \\
\hline $\begin{array}{l}\text { 101. Pyxine reticulata (Vain.) } \\
\text { Vain.** }\end{array}$ & $\mathrm{B}$ & $\mathrm{F}$ & & & & & & & + & + & + & & & & + & + & & + & & & + & \\
\hline $\begin{array}{l}\text { 102. Pyxine sorediata (Ach.) } \\
\text { Mont. }\end{array}$ & $\mathrm{B} / \mathrm{R}$ & $\mathrm{F}$ & & + & & & & & & & & & & & & & & & & & & \\
\hline $\begin{array}{l}\text { 103. Pyxine subcinerea } \text { Stirt.* } \\
\text { Pilocarpaceae }\end{array}$ & B & $\mathrm{F}$ & + & & & & + & + & + & & & + & & + & & + & & & + & + & + & \\
\hline $\begin{array}{l}\text { 104. Fellhanera bouteillei } \\
\text { (Desm.) Vězda }\end{array}$ & $\mathrm{L}$ & Fo & & & & & & & & & & & & & & & & & & & & \\
\hline $\begin{array}{l}\text { 105. Micarea sp. } \\
\text { Porpidiaceae }\end{array}$ & B & $\mathrm{C}$ & & + & & & & & & & & & & & & & & & & & & \\
\hline $\begin{array}{l}\text { 106. Mycobilimbia hunana } \\
\text { (Zahlbr.) D.D. Awasthi* }\end{array}$ & B & $\mathrm{C}$ & & & & & & & & & & & & & & & & & & & + & \\
\hline Pyrenulaceae & & & & & & & & & & & & & & & & & & & & & & \\
\hline $\begin{array}{l}\text { 107. Anthracothecium } \\
\text { platystomum Müll. Arg. }\end{array}$ & B & $\mathrm{C}$ & & + & & & & & & & & & & & & & & & & & & \\
\hline $\begin{array}{l}\text { 108. Anthracothecium } \\
\text { variolosum (Pers.) Müll. } \\
\text { Arg. }\end{array}$ & B & $\mathrm{C}$ & & & & & & & + & & & & & & & & & & & & & \\
\hline 109. Lithothelium $\mathrm{sp}$ & B & $\mathrm{C}$ & & & & & & & + & & & & & & & & & & & & & \\
\hline $\begin{array}{l}\text { 110. Pyrenula anomala (Ach.) } \\
\text { Vain* }\end{array}$ & B & $\mathrm{C}$ & & & & & & & + & & & & & & & & & & & & & \\
\hline $\begin{array}{l}\text { 111. Pyrenula astroidea (Fée) } \\
\text { R.C. Harris }\end{array}$ & B & $\mathrm{C}$ & & + & & & & & & & & & & & & & & & & & & \\
\hline $\begin{array}{l}\text { 112. Pyrenula immissa (Stirt.) } \\
\text { Zahlbr. }\end{array}$ & B & $\mathrm{C}$ & & + & & & & & & & & & & + & & + & & & & & + & \\
\hline $\begin{array}{l}\text { 113. Pyrenula interducta (Nyl.) } \\
\text { Zahlbr.* }\end{array}$ & B & $\mathrm{C}$ & & & & & & & + & & & + & & & & & & & & & & \\
\hline $\begin{array}{l}\text { 114. Pyrenula introducta (Stirt) } \\
\text { Zahlbr.* }\end{array}$ & B & $\mathrm{C}$ & & & & + & & + & & + & & & & & & + & & & + & & & + \\
\hline 115. Pyrenula leucostoma Ach. & B & $\mathrm{C}$ & & + & & & & & & & & & & & & & & + & & & & \\
\hline $\begin{array}{l}\text { 116. Pyrenula macularis } \\
\text { (Zahlbr.) R.C. Harris }\end{array}$ & $\mathrm{B}$ & $\mathrm{C}$ & & + & & & & & & & & & & & & & & + & & & & \\
\hline 117. Pyrenula pinguis Fée & B & $\mathrm{C}$ & & & & + & & + & & & + & & & & & & & + & + & + & & \\
\hline 118. Pyrenula quassiaecola Fée. & B & $\mathrm{C}$ & & + & & & & + & & & + & & & & & & & + & & & + & \\
\hline $\begin{array}{l}\text { 119. Pyrenula submastophora } \\
\text { Ajay Singh \& Upreti** }\end{array}$ & $\mathrm{B}$ & $\mathrm{C}$ & & & & & & & & & & & + & & & & & & & & & \\
\hline
\end{tabular}




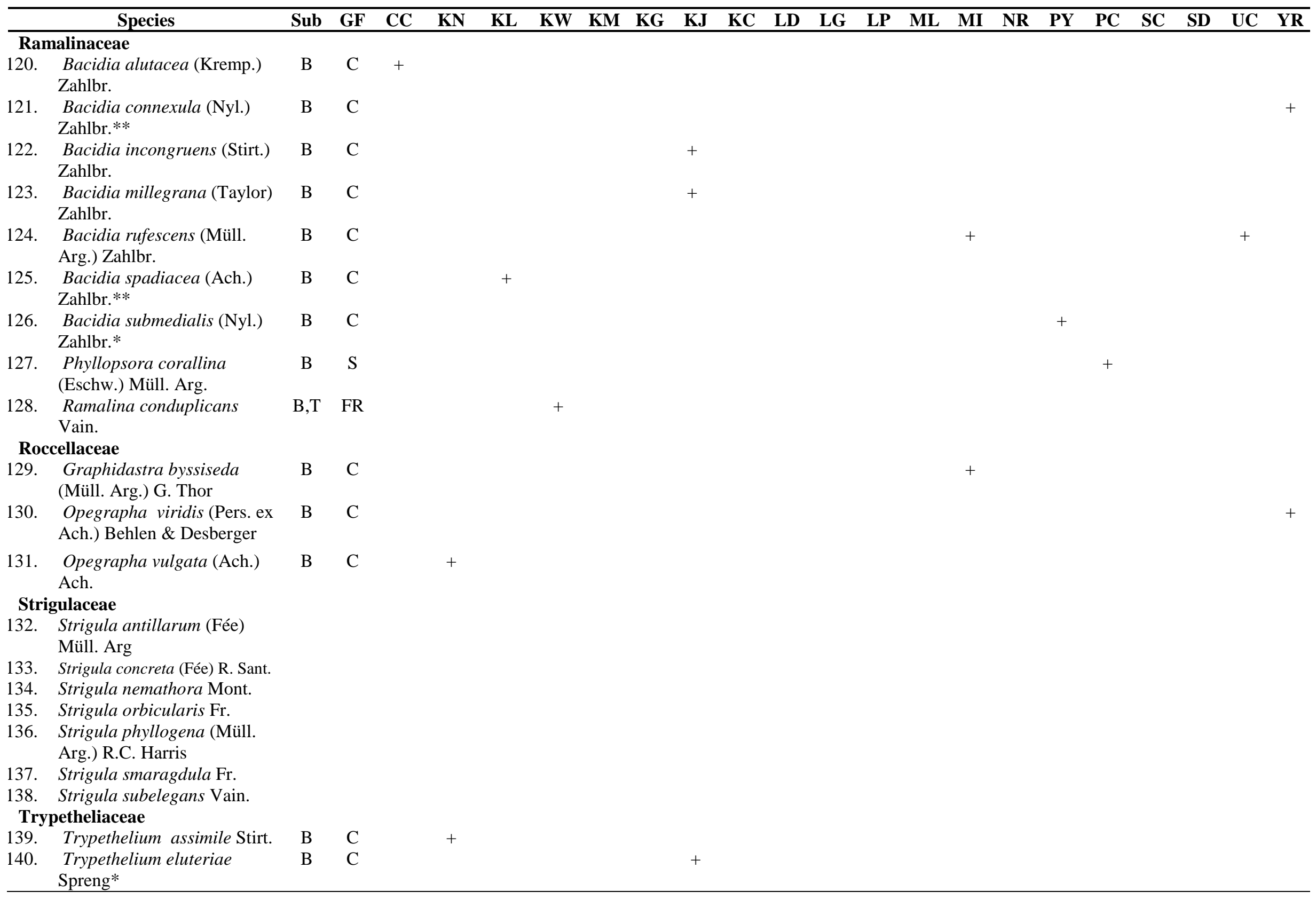


Note: Sub - Substratum, GF - Growth form, B - Bark, T - Twig, R - Rock, CW - Concrete wall, M Moss, L -Leaves, C - Crustose, F - Foliose, Fo - Foliicolous, Fr -Fruticose, D - Dimorphic, S Squamulose, Le - Leprose, Site CC - Chingkhei Ching, KN - Kaina, KL - Keibul Lamjao, KW - Keirao Wangkhem, KM - Keitelmanbi, KG - Khongampat, KJ - Konthoujam, KC - Konung Ching, LD Lamdan, LG - Langol, LP - Loukoipat, ML - Maibam Lotpa Ching, MI - Mana Ingkhol, NR - Ngariyan, PY - Phayeng, PC - Porou Ching, SC - Sadu Chiru, SD - Singda, UC - Uyok Ching, YR - Yurembam

*New addition to the lichen flora of Manipur; **New records for Northeast India.

180:60:8 ml) and solvent EA (Diethyl ether: Acetic acid: 200:2 ml). Specimens were identified by comparing the morphological and the biochemical test results with the literature and identification keys (Awasthi 2007, Divakar \& Upreti 2005). The identified specimens are preserved in the National Botanical Research Institute (NBRI) herbarium, Lucknow (LWG) and the Department of Ecology and Environmental Science, Assam University, Silchar (AUS).

\section{Results}

The composition of the lichen flora of the Manipur State was studied and species richness is expressed as alpha-diversity $(\alpha)$ totalling 140 species belonging to 50 genera and 23 families (Table 1). The crustose morphotype (49\% of the total species found) was most dominant followed by the foliose $(43 \%)$, the dimorphic, fruticose, leprose and squamulose morphotypes, each contributing $4 \%, 2 \%, 1 \%$ and $1 \%$, respectively.

Nine species belonging to a special group of phorophytic lichens that usually colonize live leaves, also known as foliicolous lichens, were also encountered. Species richness was remarkably denoted by the presence of about 75-85 foliicolous lichens colonies on a single leaf. A few foliicolous lichens were also found inhabiting the leaves of orchids and of Ferula asafoetida (Hing) planted in preservation plot for educational purposes at an ex-situ Orchid Preservation Centre, Khongampat located near the capital town of Imphal of Manipur State, Northeast India. However, these were at a very young stage and hence could not be identified.

\section{Discussion}

The occurrence of fewer fruticose lichens in the area is due relatively poor light conditions in almost all the study sites. Appropriate light conditions are a pre-requisite for luxuriant growth of foliose and fruticose lichens (James et al. 1977, Broad 1989). Wolseley \& Pryor (1999) reported that fruticose lichens are light sensitive and are typically associated with the canopy environment, explicitly twigs and branches and indeed, are often referred to as light demanding lichens.

Based on the annotated checklist of Singh \& Sinha (2010) and recent publications on new records of lichens of Northeast India (Rout et al. 2010, Daimari et al. 2014, Devi et al. 2013; Upreti et al. 2014, Logesh et al. 2015), 39 species belonging to 21 genera and 14 families have been listed as new addition to lichen flora of Manipur. Of these, 14 species are new to Northeast India.

Phytogeographic affinities and distribution of the newly recorded lichens in Manipur is also furnished.

\section{Taxonomy}

\section{CANDELARIACEAE}

1. Candelaria concolor (Dicks.) Stein, Cohn, Krypt. Fl. Schles. 2(2): $84.1879 \quad$ Fig. 2

$=$ Lichen concolor Dicks., Fasc. Pl. Crypt. Br.: Pl. 9, fig. 8. 1783.

Distribution $-C$. concolor is a new report to Manipur and is previously known from

Himachal Pradesh, Jammu \& Kashmir, Karnataka, Nagaland, Sikkim and Uttarakhand.

Specimens examined - India, Manipur, Imphal West, Yurembam, 736 m, 2 Nov 2011, RKSD, 139/A (AUS). 


\section{CHRYSOTHRICACEAE}

2. Chrysothrix candelaris (L.) J.R. Laundon, Lichenologist 13(2): 101- 121, 1981

= Byssus candelaris L., Sp. PL 2: 1169, 1753.

Distribution $-C$. candelaris is a new report to Manipur and is previously reported from

Assam, Himachal Pradesh, Jammu \& Kashmir, Sikkim and Tamil Nadu of India.

Specimens examined - India, Manipur, Bishnupur district, Keibul Lamjao, 911 m, 11 Feb 2015, RKSD, 212 (AUS).

\section{CLADONIACEAE}

3. Cladonia awasthiana Ashti \& Upreti, Biblioth. Lichenol. 88: 9. 2004.

Distribution - C. awasthiana is widely distributed in Arunachal Pradesh, Himachal Pradesh,

Jammu \& Kashmir, Sikkim and Uttarakhand, and is a new report to Manipur.

Specimens examined - India, Manipur, Imphal West, Phayeng, 871 m, 2 Jan 2012, RKSD, 27097 (LWG/AUS).

4. Cladonia macroceras (Delise) Hav., Bergens Mus. Aarbok. Naturgidensk. Rekke 1927 (3): 12. 1928.

\section{Fig. 3}

=Cenomyce gracilis var. macroceras Delise in Duby, Bot. Gall. 2: 624. 1830 .

Distribution - In India, the species was previously reported from Uttarakhand. The species is a terricolous lichen growing on red loam mixed with gravels, and is a new report to Manipur.

Specimen examined - INDIA, Manipur, Imphal West district, Khongampat, 816 m, 10 Dec 2012, AUSJR-RKSD167 (LWG/AUS).

5. Cladonia rei Schaer., Lich. Helv. Spic.: 34, 1823.

Distribution $-C$. rei is a new report to Manipur and was previously reported from Arunachal Pradesh, Himachal Pradesh, Jammu \& Kashmir and Uttarakhand.

Specimens examined - India, Manipur, Imphal West, Mana Ingkol Ching, 816 m, 28 Dec 2011, RKSD, 27050 (LWG).

\section{COLLEMATACEAE}

6. Collema leptaleum var. biliosum (Mont.) Dengel., Symb. Bot. Upsal. 20(2): 105. 1974.

Fig. 4

= Collema nigrescens var. biliosum Mont., Ann. Sci. Nat. Bot., ser. 2(18): 20, 1842.

Distribution $-C$. leptaleum var. biliosum was previously reported from Maharashtra, Tamil Nadu and Uttarakhand, and is a new report for Northeast India.

Specimens examined - India, Manipur, Senapati, Sadu Chiru, 1066 m, 8 Aug 2013, RKSD, 14-3-025713 (LWG/AUS).

7. Leptogium pichneum (Ach.) Malme, Ark. Bot. 19(8): 20, 1924.

Fig. 5

Distribution - L. pichneum was previously reported from Andaman \& Nicobar Islands, Kerala, Madhya Pradesh and Tamil Nadu, and is a new report for Northeast India.

Specimens examined - India, Manipur, Imphal West, Konthoujam Lairembi, 763 m, 24 Mar 2012, RKSD, 439 (AUS).

8. Leptogium pseudopapillosum P.M. Jørg. Symb. Bot. Upsal., 32(1): 120, 1997.

Distribution - L. pseudopapillosum was previously reported from Kerala, Nagaland, Tamil Nadu and Uttarakhand, and is a new report to Manipur.

Specimens examined - India, Manipur, Senapati, Sadu Chiru, 1066 m, 24 Mar 2015, RKSD, 14-3-025715 (LWG).

\section{GRAPHIDACEAE}

9. Graphis caesiella Vain, Acta Soc. Fauna Fl. Finn. 7: 122, 1890.

Distribution $-G$. caesiella was previously reported only from Lakshadweep and is a new report to Manipur.

Specimens examined - India, Manipur, Bishnupur, Loukoipat, 854 m, 11 Feb 2015, RKSD, 1213 (AUS). 
10. Graphis pyrrhocheiloides Zahlbr., Cat. Lich. Univ. 2: 321, 1923.

Fig. 6

Distribution - G. pyrrhocheiloides is a new report for Northeast India and was previously reported from Karnataka, Maharashtra, and West Bengal.

Specimens examined - India, Manipur, Bishnupur, Loukoipat, 854 m, 11 Feb 2015, RKSD, 14-3-025714 (AUS).

\section{PORPIDIACEAE}

11. Mycobilimbia hunana (Zahlbr.) D.D. Awasthi \& R. Mathur, Proc. Indian Acad. Sci., Pl. Sci. 97(6): 501, 1987.

= Bacidia hunana Zahlbr. in Handel- Mazzetti, Symb. Sinic. Pars III: 113, 1930.

Distribution $-M$. hunana is reported from Mizoram, Nagaland and West Bengal, and is a new report to Manipur.

Specimens examined - India, Manipur, Thoubal, Uyok Ching, 819 m, 8 Aug 2013, RKSD, 15-25680 (LWG/AUS).

\section{NEPHROMATACEAE}

12. Nephroma sikkimense Asahina, J. Jap. Bot. 38: 193.

Fig. 7

Distribution $-N$. Sikkimense is an endemic species which was reported only from Sikkim.

Specimens examined - India, Manipur, Senapati, Sadu Chiru, 1066 m, 8 Aug 2013, RKSD, 15-25672 (LWG/AUS).

\section{PARMELIACEAE}

13. Bulbothrix tabacina (Mont. \& Bosch.) Hale, Phytologia 28: 481.1974 \& Smithsonian Contr. Bot. 32: 24, 1976.

= Parmelia tabacina Mont. \& Bosch, Syll. Gen. Sp. Crypt.: 327, 1856.

Distribution $-B$. tabacina is a new report to Manipur. The species is distributed only in Nagaland.

Specimens examined - India, Manipur, Imphal East district, Chingkhei Ching, $867 \mathrm{~m}$, 18.10.2011, RKSD, 27035 (LWG); Imphal West, Mana Ingkol Ching, 816 m, 28 Dec 2011, RKSD, 311 (AUS).

14. Hypotrachyna revoluta (Florke) Hale, Smithsonian Contr. Bot. 25: 60. 1975.

= Parmelia revoluta Flörke, Deutschl. Lich. 1: 11. 1815.

Fig. 8

Distribution - The species earlier is reported only from Tamil Nadu. H. revoluta is a new report for Northeast India.

Specimens examined - India, Manipur, Imphal East, Keirao Wangkhem, 834 m, 18 Feb 2012, RKSD, 383 (AUS).

15. Parmotrema andinum (Müll. Arg.) Hale, Phytologia 28: 334, 1974

Fig. 9

= Parmelia andina Müll. Arg., Rev. Mycol. (Toulouse) 1: 169. 1879.

Distribution - This species is known from Andhra Pradesh, Himachal Pradesh, Jharkhand, Karnataka, Madhya Pradesh, Orissa, Tamil Nadu and Uttarakhand, and is a new report for Northeast India.

Specimens examined - India, Manipur, Imphal West, Langol, 811 m, 13 Oct 2011, RKSD, 27061(LWG); Konthoujam Lairembi, 763 m, 18 Feb 2012, RKSD, 415(AUS).

16. Parmotrema crinitum (Ach.) M. Choisy, Bull. Mens. Soc. Linn. Soc. Bot. Lyon 21: 175, 1952. = Parmelia crinita Ach., Syn. Meth. Lich.: 196, 1814.

Distribution - A cosmopolitan species known from Andhra Pradesh, Himachal Pradesh, Jammu \& Kashmir, Karnataka, Kerala, Tamil Nadu and Uttarakhand, but is newly reported in Manipur.

Specimens examined - INDIA: Manipur, Imphal East, Keirao Wangkhem, 837 m, 9 Oct 2011, RKSD, 01(AUS); Imphal West, Khongampat, 799 m, 10 Dec 2011, RKSD, 27057 (LWG). 
17. Parmotrema eunetum (Stirt.) Hale, Phytologia 28: 336, 1974.

Fig. 10

= Parmelia euneta Stirt., Scott. Naturalist (Perth) 4: 298, 1878.

Distribution - The species is a new report for Northeast India being previously known for Kerala and Uttarakhand.

Specimens examined - India, Manipur, Imphal West, Konthoujam Lairembi, 763 m, 18 Feb 2012, RKSD, 27056 (LWG); Bishnupur, Ngariyan Hills, 848 m, 12 Dec 2011, RKSD, 211(AUS).

18. Parmotrema indicum Hale, Mycotaxon 5: 436, 1977.

Distribution $-P$. indicum is new report for Northeast India. It was previously reported for Kerala and Tamil Nadu.

Specimens examined - India, Manipur, Imphal West, Langol, elevation $811 \mathrm{~m}, 13$ Oct 2011, RKSD, 27060 (LWG); Imphal East district, Chingkhei Ching, elevation 811 m, 18 Oct 2011, RKSD 78/B(AUS).

19. Parmotrema mesotropum (Mull. Arg.) Hale, Phytologia 28: 337, 1974.

= Parmelia mesotropa Müll. Arg., Rev. Mycol. (Toulouse) 10: 55, 1888.

Distribution - P. mesotropum is reported from Arunachal Pradesh, Himachal Pradesh, Karnataka, Kerala, Madhya Pradesh and Uttarakhand, and is new addition to the lichen flora of Manipur.

Specimens examined - India, Manipur, Imphal West, Phayeng, elevation $871 \mathrm{~m}, 02$ Jan 2012, RKSD, 375 (AUS).

20. Parmotrema saccatilobum (Taylor) Hale, Phytologia 28: 339, 1974.

= Parmelia saccatiloba Taylor in Hook.f., London J. Bot. 6: 174, 1847.

Distribution - The taxon is widely distributed in Andaman \& Nicobar Islands, Assam, Goa, Kerala, Maharashtra, Nagaland, Sikkim, Tamil Nadu, Uttarakhand and West Bengal, and is new report to Manipur.

Specimens examined - India, Manipur, Imphal West, Mana Ingkol Ching, 816 m, 23 Oct 2011, RKSD, 27059 (LWG); Imphal West, Phayeng, elevation 871 m, 02 Jan 2012, RKSD, 367 (AUS).

21. Usnea pangiana Stirt., Scott. Naturalist (Perth) 7: 77, 1883.

Distribution - U. pangiana is reported from Arunachal Pradesh, Assam, Kerala, Meghalaya, Nagaland, Sikkim, Uttarakhand and West Bengal, and is new report to Manipur.

Specimens examined - India, Manipur, Churachandpur, Lamdan, 1102 m, 4 Jun 2014, RKSD, 15-25677 (LWG/AUS).

\section{PERTUSARIACEAE}

22. Pertusaria tetrathalmia (Fée) Nyl., Acta Soc. Sci. Fenn. 7: 448, 1863.

= Trypethelium tetrathalmium Fée, Essai Crypt. Ecorc, 69, 1824.

Distribution $-P$. tetrathalmia is new report for Northeast India and it was previously reported for Karnataka, Kerala, Madhya Pradesh and Tamil Nadu.

Specimens examined - India, Imphal West district, Yurembam, elevation $736 \mathrm{~m}, 2$ Nov 2011, RKSD, 144 (AUS).

\section{PHYSCIACEAE}

23. Dirinaria applanata (Fee) D.D.Awasthi., J. Indian Bot. Soc. 49: 135, 1970

= Parmelia applanata Fée, Essai Crypt. Ecorc.: 126, 1824.

Distribution - D. applanata is widely distributed in Andaman \& Nicobar Islands, Karnataka, Madhya Pradesh, Maharashtra, Nagaland, Sikkim, Tamil Nadu, Uttar Pradesh, Uttarakhand and West Bengal and is new report to Manipur.

Specimens examined - India, Manipur, Imphal East district, Kaina, 907 m, 24 Mar 2012, RKSD 27064 (LWG); Imphal West, Mana Ingkol Ching, 816 m, 28 Dec 2011, RKSD, 311 (AUS).

24. Dirinaria confluens (Fr.) D.D. Awasthi, Biblioth. Lichenol. 2: 28. 1975.

$=$ Parmelia confluens Fr., Syst. Orb. Veg. 1:284. 1825. 
Distribution - D. confluens is widely distributed in Andaman \& Nicobar Islands, Arunachal Pradesh, Karnataka, Kerala, Madhya Pradesh, Maharashtra, Nagaland, Orissa, Sikkim, Tamil Nadu and Uttar Pradesh and is new report to Manipur.

Specimens examined - India, Manipur, Imphal West, Mana Ingkol Ching, 816 m, 23 Oct 2011, RKSD, 108 (AUS).

25. Dirinaria picta (Sw.) Clem. \& Shaer., Gen. Fungi : 323, 1931

= Lichen pictus Sw., Prodr.: 146, 1788.

Distribution - D. picta is reported for Andaman \& Nicobar Islands, Arunachal Pradesh, Assam, Lakshadweep, Tamil Nadu, Uttar Pradesh and West Bengal and is new report to Manipur.

Specimens examined - India, Manipur, Imphal East district, Porou Ching, 801 m, 24 Mar 2012, RKSD, 485 (AUS).

26. Heteterodermia albidiflava (Kurok.) D.D.Awasthi, Geophytology 3: 113, 1973; Anaptychia albidiflava Kurok., Beih. Nova Hedwigia 6: 42, 1962.

Distribution - An endemic species to India, known for Himachal Pradesh, Madhya Pradesh, Mizoram, Sikkim and West Bengal, and is new addition to the lichen flora of Manipur.

Specimens examined - India, Manipur, Imphal East district, Keirao Wangkhem, 834 m, 18 Feb 2012, RKSD, 404 (AUS).

27. Heterodermia hypochraea (Vain.) Swinscow \& Krog, Lichenologist 8: 119. 1976. to Manipur.

Distribution $-H$. hypochraea was previously reported only for Nagaland, and is new report

Specimens examined - India, Manipur, Imphal East district, Keirao Wangkhem, 834 m, 18 Feb 2012, RKSD, 04 (AUS).

28. Phaeophyscia endococcina (Körb.) Moberg, Symb. Bot. Upsal 22(1): 35, 1977

= Parmelia endococcina Körb., Parerga Lichenol: 36, 1859.

Distribution $-P$. endococcina is previously known from Arunachal Pradesh, Himachal Pradesh, Jammu \& Kashmir, Madhya Pradesh, Maharashtra, Sikkim and Uttarakhand, and is a new addition to the lichen flora of Manipur.

Specimens examined - India, Manipur, Senapati, Sadu Chiru, 1066 m, 8 Aug 2013, RKSD, 14-3-025727 (LWG/AUS).

29. Pyxine himalayensis D.D. Awasthi, Phytomorphology 30: 371, 1980.

Fig. 11

Distribution $-P$. himalayensis is previously reported for West Bengal. This species is new report for Northeast India

Specimens examined - India, Manipur, Imphal East, Keirao Wangkhem, 837 m, 9 Oct 2011, RKSD, 24/A (AUS).

30. Pyxine petricola Nyl., J. Bot. London 14: 263, 1876.

Distribution $-P$. petricola is widely distributed in Assam, Himachal Pradesh, Jammu \& Kashmir, Jharkhand, Karnataka, Kerala, Madhya Pradesh, Maharashtra and Tamil Nadu and is new report to Manipur.

Specimens examined - India, Manipur, Imphal West, Phayeng, 871 m, 2 Jan 2012, RKSD, 354 (AUS).

31. Pyxine reticulata (Vain.) Vain., Ann. Acad. Sci. Fenn., ser. A, 70, 1914.

= Physcia reticulate Vain. In Hiern \& al., Cat. Afr. Pl. 2(2): 412, 1901.

Distribution - A cosmopolitan species previously reported for Andaman \& Nicobar Islands, Karnataka and Tamil Nadu. P. reticulata is new report for Northeast India.

Specimens examined - India, Manipur, Imphal West, Mana Ingkol Ching, 816 m, 23 Oct 2011, RKSD, 98 (AUS); Imphal West, Khongampat, 799 m, 10 Dec 2011, RKSD, 190 (AUS).

32. Pyxine subcinerea Stirt., Trans. \& Proc. New Zealand Inst. 30: 397, 1898.

Distribution - A cosmopolitan species known from Himachal Pradesh, Jammu \& Kashmir, Madhya Pradesh, Nagaland, Sikkim, Tamil Nadu, Uttarakhand, and West Bengal. P. subcinerea is a new report to Manipur. 

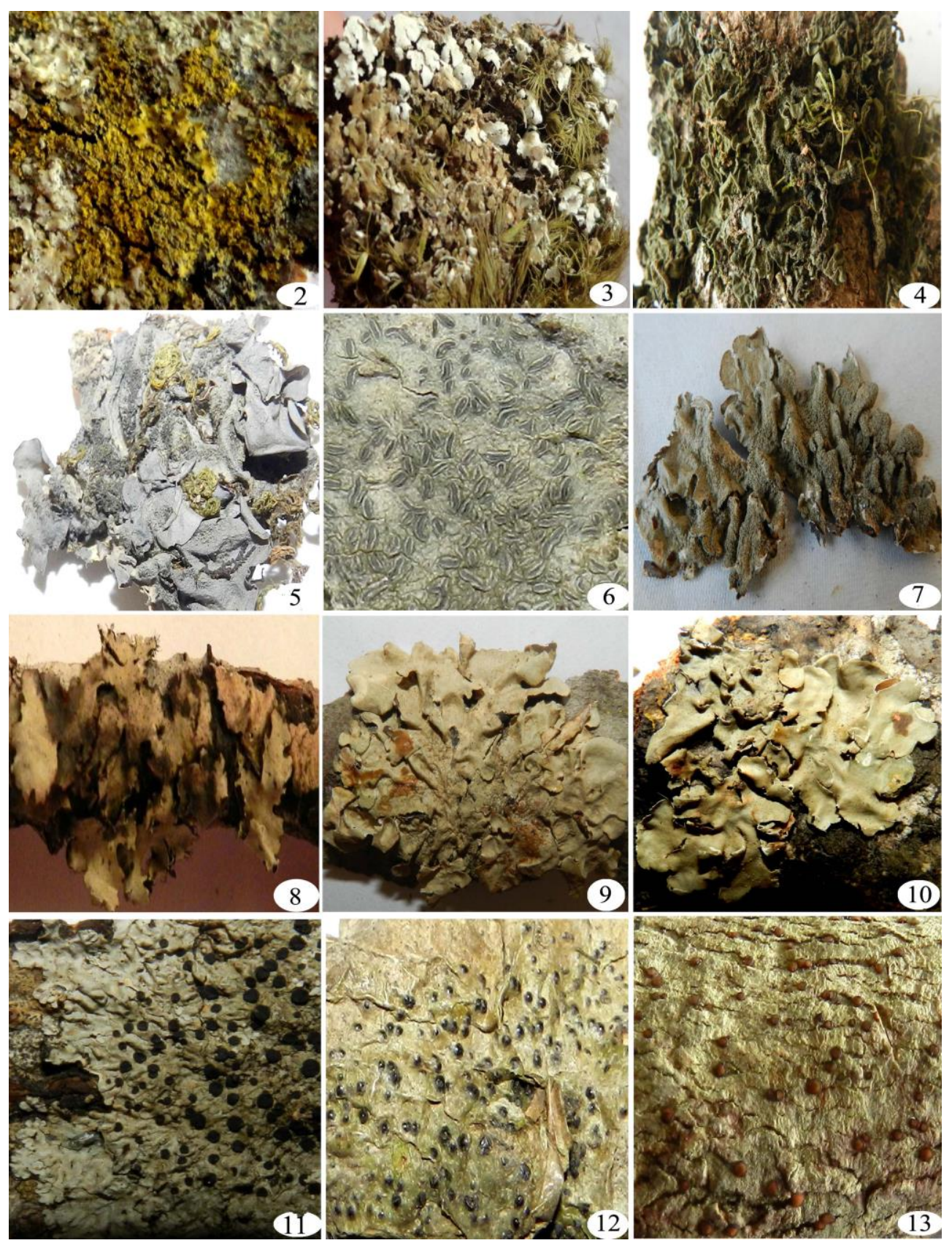

Figs 2-12-New reports of lichens of Manipur state. 2 Candelaria concolor (Dicks.) Stein 3 Cladonia macroceras (Delise) Hav. 4 Collema leptaleum var. biliosum (Mont.) Dengel. 5 Leptogium pichneum (Ach.) Malme. 6 Graphis pyrrhocheiloides Zahlbr. 7 Nephroma sikkimense Asahina. 8 Hypotrachyna revoluta (Florke) Hale. 9 Parmotrema andinum (Müll. Arg.) Hale. 10 Parmotrema eunetum (Stirt.) Hale. 11 Pyxine himalayensis D.D. Awasthi. 12 Pyrenula submastophora Ajay Singh and Upreti. 13 Bacidia connexula (Nyl.) Zahlbr.

Specimens examined - India, Manipur, Bishnupur, Ngariyan hills, 992 m, 12 Dec 2011, RKSD, 258 (AUS).

\section{PYRENULACEAE}

33. Pyrenula anomala (Ach.) Vain., Ann. Acad. Sci. Fenn., ser. A, 6: 189, 1915.

= Trypethelium anomalum Ach., Syn. Meth. Lich.: 105, 1814.

Distribution - The species is new report to Manipur and was previously reported for Andaman \& Nicobar Islands and Arunachal Pradesh.

Specimens examined - India, Manipur, Imphal West, Konthoujam Lairembi, 763 m, 24 Mar 2012, RKSD, 15-25676 (LWG). 
34. Pyrenula introducta (Stirt) Zahlbr., Cat. Lich. Univ, 1: 433, 1922.

= Verrucaria introducta, Proc. Roy. Soc. Glasgow, 13: 191, 1881.

Distribution $-P$. introducta is previously reported for Andaman \& Nicobar Islands, Andhra Pradesh, Assam, Arunachal Pradesh, Kerala, Sikkim, Tamil Nadu, Uttar Pradesh and West Bengal, and is new report for Manipur.

Specimens examined - India, Manipur, Imphal West, Khongampat, 856 m, 10 Dec 2011, RKSD, 176 (LWG); Imphal East, Keirao Wangkhem, 834 m, 18 Feb 2012, RKSD, 16 (AUS).

35. Pyrenula submastophora Ajay Singh and Upreti, Geophytology 17: 85, 1987 . Fig. 12

Distribution - The species is new report for Northeast India and was previously known for Andaman \& Nicobar Islands and Kerala.

Specimens examined - India, Manipur, Bishnupur, Loukoipat, 854 m, 11 Feb 2015, RKSD, 213 (AUS).

\section{RAMALINACEAE}

36. Bacidia connexula (Nyl.) Zahlbr., Cat. Lich. Univ. 4:187, 1926

$=$ Lecidea connexula Nyl., Lich. Jap.: 111, 1890.

Fig. 13

Distribution $-B$. connexula is new report for Northeast India. It was previously reported for Madhya Pradesh and West Bengal.

Specimens examined - India, Imphal West district, Yurembam, elevation 736 m, 2 Nov 2011, RKSD, 144 (AUS).

37. Bacidia spadiacea (Ach.) Zahlbr., Denkschr. Kaiserl. Akad. Wiss. Wien. Math. Naturwiss. Kl. 83:128, 1909

=Lecidea spadicea Ach., Synops. Lich.: 34, 1814.

Distribution $-B$. spadicea is a new report for Northeast India. This species is reported from Tamil Nadu and West Bengal.

Specimens examined - India, Manipur, Bishnupur district, Keibul Lamjao, 891 m, 2 Feb 2015, RKSD, 1167 (AUS).

38. Bacidia submedialis (Nyl.) Zahlbr., Cat. Lich. Univ. 4:243, 1926

= Lecidea submedialis Nyl., Acta Soc. Sci. Fenn. 26(10): 14, 1900.

Distribution - This species is a new report for Manipur. Previously it was reported for Arunachal Pradesh, Goa, Karnataka, Maharashtra, Tamil Nadu, Uttar Pradesh, and West Bengal.

Specimens examined - India, Manipur, Imphal West, district, Phayeng, 871 m, 2 Jan 2012, RKSD, 350 (AUS).

\section{TRYPETHELIACEAE}

39. Trypethelium eluteriae Spreng, Anleit, Kent. 3:351, 1804.

Distribution $-T$. eluteriae is reported as a new addition to the lichen flora of Manipur. Previously it was reported for Andaman \& Nicobar Islands, Arunachal Pradesh, Karnataka, Kerala, Madhya Pradesh, Maharashtra, Meghalaya, Nagaland, and Tamil Nadu.

Specimens examined - India, Manipur, Imphal West, Konthoujam Lairembi, 763 m, 24 Mar 2015, RKSD, 15-25675 (LWG).

\section{Acknowledgements}

The first author (RKSD) is thankful to University Grants Commission (UGC), New Delhi, India for financial support. The authors also thank the Director, CSIR National Botanical Research Institute (NBRI), Lucknow, India and P.G. Department of Botany, D.M. College of Science, Imphal, Manipur for providing laboratory facilities.

\section{References}

Awasthi DD. 1960 - Contributions to the lichen flora of India and Nepal. I. The genus Physcia (Ach.) Vain. Journal of Indian Botanical Society 39, 1-21. 
Awasthi DD. 1987 - A new position for Platysma thomnosii, Stirton. Journal of the Hattori Botanical Laboratory 63, 367-372.

Awasthi DD. 1988 - A key to the Macrolichens of India and Nepal, Journal of the Hattori Botanical Laboratory 65, 207-302.

Awasthi DD. 2007 - A Compendium of the Macrolichens from India, Nepal and Sri Lanka. Bishen Singh Mahendra Pal Singh, Dehra Dun.

Broad K. 1989 - Lichens in southern woodlands. Forest Officer, Forestry Commission, Handbook 4, London: Her Majesty's stationery office.

Daimari R, Hazarika N, Hoque RR, Nayaka S, Upreti, DK. 2014 - New records of epiphytic lichens from three districts of Assam, India. Indian Forester, 140(8), 807-811.

Devi RKS, Rout J, Upreti DK, Pinokiyo A. 2013 - New additions to the microlichens of Manipur, North East India. Phytotaxonomy. 13, 75-83.

Divakar PK, Upreti DK. 2005 - Parmeloid lichens in India, Bishen Singh and Mahendra Pal Singh, Dehradun, India. p. 1-448.

Groombridge B. 1992 - Global biodiversity: Status of the earth's living resources, Chapman and Hall.

James PW, Hawksworth DL, Rose F. 1977 - Lichen communities in the British Isles: a preliminary conspectus in Seaward, M.R.D. (eds). Lichen Ecology, London Academic Press. P. 295413.

Logesh A, Chinlampianga M, Shukla A, Upreti DK. 2015 - Studies on Lichens of Mizoram, Northeast India. Proceedings of the National Academy of Sciences, India Section B: Biological Sciences 1-13.

Müll. Arg. 1892 - Lichenes Manipuriensis a.cl. Dr. G. Watt circa Manipur ad limites orientalis Indiae Orientalis 1881-82 lecti. Journal of the Linnean Society, Botany 29, 217-231.

Patwardhan, Nagarkar - 1982. Three interesting species of macrolichens from Northeast India. Current Science 51(10), 527-528.

Rout J, Das P, Upreti DK. 2010 - Epiphytic lichen diversity in a Reserve Forest in southern Assam, northeast India. Tropical Ecology 51, 281-288.

Singh A. 1984 - The lichen genus Anthracothecium from Manipur, India. Geophytology. 14, 6973.

Singh KP. 1980 - Awasthiella a new lichen genus from Manipur, India. Norwegian Journal of Botany 27, 33-35.

Singh KP. 1981a - Microlichens from Manipur, India. Geophytology. 11(2), 242-256.

Singh KP. 1981b - Macrolichens from Manipur, India. Biological Memoirs 6(2), 145-168.

Singh KP. 1983 - Catillaria manipuriensis, a new species of lichens and a note on Lopadium austroindicum from India. Current Science 52, 165-166.

Singh KP. 1984 - On the species of Buellia and Diplotomma from Manipur India. Bulletin Botanical Survey of India 26(1-2), 62-64.

Singh KP, Pinokiyo A. 2003 - Foliicolous lichens and their diversity in North East India. Procedings of National Academy of Science India, B 73(II):177-185.

Singh KP and Sinha GP. 2010 - Indian lichens: An annotated checklist. Botanical Survey of India. p.1-507.

Singh KP, Upreti DK. 1986 - On the species of of Cladonia from Arunachal Pradesh and Manipur, India. Phytotaxonomy. 6, 84-92.

Upreti DK. 1990 - Lichen genus Pyrenula in India Pyrenula subducta spore type. Journal of Hattori Botanical Laboratory 68, 269-278.

Upreti DK. 1993. - Lichen genus Pyrenula from India: The species with spores of Pyrenula brunnea type. Bulletin de la Société Botanique de France, Lettres Botaniques 138(3), 241247.

Upreti DK, Debnath R, Uppadhyay V, Rout J. 2014 - Diversity and distribution of lichens in north and west districts of Tripura. Phytotaxonomy, 14, 122-129. 
Walker FJ, James PW. 1980 - A revised guide and microchemical techniques for the identification of lichen substances. Bulletin British Lichenological Society 46, 13-29.

Wolseley PA, Pryor KV. 1999 - The lichens of epiphytic twig communities on Quercus petrarea in a Welsh Woodland site (Tycanal) fir evaluating environmental changes. Lichenologist. 31 (1), 41-61. 